\title{
Quantum Phase Interference for Quantum Tunneling in Spin Systems
}

\author{
J.-Q. Liang ${ }^{1,2 *}$, H. J. W. Müller-Kirsten ${ }^{1 \dagger}$, D. K. Park ${ }^{1,3 \ddagger}$ and F.-C. $\mathrm{Pu}^{4}$ \\ 1. Department of Physics, University of Kaiserslautern, D-67653 Kaiserslautern, Germany \\ 2. Institute of Theoretical Physics and Department of Physics, Shanxi University, Taiyuan, \\ Shanxi 030006, People's Republic of China \\ 3. Department of Physics, Kyungnam University, Masan, 631-701, Korea \\ 4. Institute of Physics and Center for Condensed Matter Physics, Chinese Academy of Sciences, \\ Beijing 100080, People's Republic of China and Department of Physics, Guangzhou Normal \\ College, Guangzhou 510400, People's Republic of China
}

\begin{abstract}
The point-particle-like Hamiltonian of a biaxial spin particle with external magnetic field along the hard axis is obtained in terms of the potential field description of spin systems with exact spin-coordinate correspondence. The Zeeman energy term turns out to be an effective gauge potential which leads to a nonintegrable phase of the Euclidean Feynman propagator. The phase interference between clockwise and anticlockwise under barrier propagations is recognized explicitly as the Aharonov-Bohm effect. An additional phase which is significant for quantum phase interference is discovered with the quantum theory of spin systems besides the known phase obtained with the semiclassical treatment of spin. We also show the energy dependence of the
\end{abstract}

\footnotetext{
*e-mail:jqliang@mail.sxu.edu.cn

$\dagger$ †-mail: mueller1@pysik.uni-kl.de

‡e-mail:dkpark@genphys.kyungnam.ac.kr
} 
effect and obtain the tunneling splitting at excited states with the help of periodic instantons.

PACS numbers 75.10.Jm, 75.30.Gw, 03.65.Sq, 75.45.+j, 11.10.Ef

\section{INTRODUCTION}

Quantum tunneling in spin systems has attracted considerable attention both theoretically and experimentally in view of a possible experimental test of the tunneling effect for mesoscopic single domain particles in which case it is known as macroscopic quantum tunneling [1,2]. In particular the coherent tunneling between two degenerate metastable orientations of magnetization results in the superposition of macroscopically distinguishable (classically degenerate) states, the understanding of which is a longstanding problem in quantum mechanics and is called macroscopic quantum coherence (MQC) [3]. Till now only magnetic molecular clusters have been the most promising candidates to observe MQC [4]. Quenching of MQC for half-integer spin is a beautiful observation of quantum tunneling in spin particles and has been investigated in the literature by means of the phase interference of spin coherent state-Feynman-paths which possess a phase with obvious geometric meaning [2, 5] [ The quenching of MQC has been interpreted physically as Kramers' degeneracy. The geometric phase has also been shown to be equivalent to a Wess-Zumino type interaction in quantum mechanics [9]. However the effect of geometric phase interference is far richer than Kramers' degeneracy. For example, the Zeeman energy of an external magnetic field applied along the hard axis for a biaxial spin particle can be introduced to produce an additional geometric phase [10]. The resulting quenching of the tunneling splitting or MQC is in this case not related to Kramers' degeneracy since the external magnetic field breaks the time reversal symmetry. A more detailed investigation of quantum phase interference has been given recently [11]. An experimental observation of the magnetic field dependent oscillation of tunneling splitting has also been reported [4] for the octanuclear iron oxohydroxo cluster $\mathrm{Fe}_{8}$. The giant spin model we consider here is suitable to describe the $F e_{8}$ 
molecular cluster and therefore has practical interest.

In the traditional theory, the quantum phase induced by the Zeeman term has been investigated with the semiclassical method in which the spin is treated as a classical vector. With the help of the spin coherent state-path-integral technique an effective Hamiltonian and Lagrangian are obtained. The Zeeman term is proportional to the linear velocity and therefore emerges as a phase of the Feynman kernel in the imaginary time for quantum tunneling. It is, no doubt, interesting to explore the underlying physics of the phase related to the Zeeman energy of the magnetic field and to present an analysis of the quantum phase based on a full quantum mechanical theory of the spin system. To this end we use a recently developed method, namely, the potential field description of quantum spin systems of Ulyanov and Zaslavskii (UZ) [12] and begin with the Schrödinger equation of the spin particle. In the UZ method the spin-coordinate correspondence is exact unlike the semiclassical approach of spin where the correspondence is approximate in the large spin limit (see Appendix 1 for details). A point-particle-like Hamiltonian is obtained and the Zeeman term of the magnetic field along the hard axis of the biaxial spin system becomes a gauge potential which does not affect the equation of motion. However, the nonintegrable phase of the gauge potential leads to the quantum phase interference known as the Aharonov-Bohm(AB) effect. A substantial result derived from the quantum theory of spin is the additional phase induced by the Zeeman term which is significant to the quantum phase interference and is overlooked in the semiclassical approach. The tunneling splittings for both ground state and excited states are also obtained up to the one loop approximation. The paper is organized as follows. In Sec. 2 we first give a brief review of the semiclassical treatment of spin in quantum tunneling. The effective Hamiltonian like that of a point particle for a biaxial spin particle is obtained by starting from the Schrödinger equation following the potential field description of quantum spin systems. We show how the Zeeman energy term of the magnetic filed along hard axis becomes a gauge potential. The tunneling splitting and its oscillation with respect to the magnetic field are discussed in Sec. 3. We obtain in Sec. 4 the periodic instantons and oscillation of tunneling splitting at excited states and demonstrate the energy dependence 
of the oscillation. Our conclusions and discussions are given in Sec. 5 .

\section{EFFECTIVE POTENTIALS OF THE BIAXIAL SPIN PARTICLE IN A MAGNETIC FIELD}

We consider a giant spin model which is assumed to have biaxial anisotropy with XOY easy plane and the easy $\mathrm{y}$-axis in the XOY-plane. An external magnetic field is applied along the hard z-axis. The Hamilton operator of the model can be written as

$$
\hat{H}=K_{1} \hat{S}_{z}^{2}+K_{2} \hat{S}_{x}^{2}-g \mu_{B} h \hat{S}_{z}
$$

where $\hat{S}_{x}, \hat{S}_{y}$ and $\hat{S}_{z}$ are the three components of the spin operator. $K_{1}$ and $K_{2}$ with $K_{1}>K_{2}>0$ are the anisotropy constants and $\mu_{B}$ is the Bohr magneton. $g$ is the spin $g$-factor which is taken to be 2 here. The last term of the Hamiltonian is the Zeeman energy associated with the magnetic field h. The Hamiltonian eq.(1) is believed to describe the $F e_{8}$ molecular cluster and is the same as that in ref.[4]. Before we begin with the investigation with the UZ method we give a brief review of the semiclassical approach for the model of eq.(1). In the semiclassical method the spin is treated as a classical vector

$$
\mathbf{S}=s(\sin \theta \cos \phi, \sin \theta \sin \phi, \cos \theta)
$$

The spin-coordinate correspondence is given by the definition of canonical variables |13] $\phi$ and $p=s \cos \theta$. As shown in Appendix 1 the usual spin commutation relation $\left[\hat{S}_{i}, \hat{S}_{j}\right]=$

$i \epsilon_{i j k} \hat{S}_{k}$ is only approximately recovered in the large spin s limit. With the spin coherent state path integral technique one obtains an effective Hamiltonian [8, 11, 14]

$$
H_{s}=\frac{p^{2}}{2 m(\phi)}-\alpha p+V(\phi)
$$

with

$$
m(\phi)=\frac{1}{2 K_{1}\left(1-\lambda \sin ^{2} \phi\right)}, \quad V(\phi)=K_{2} s^{2} \sin ^{2} \phi, \quad \lambda=\frac{K_{2}}{K_{1}}, \quad \alpha=g \mu_{B} h
$$


and Lagrangian

$$
L_{s}=\frac{m(\phi)}{2}(\dot{\phi}+\alpha)^{2}-V(\phi)
$$

The position dependent mass may create an ordering ambiguity upon quantization. This is the reason why we use the elliptic integral transformation in the following to obtain a pointparticle-like Hamiltonian with a constant mass. In the above derivation we have shifted the angle $\phi$ by $\frac{\pi}{2}$ for our convenience. The periodic potential $V(\phi)$ has degenerate vacua. The quantum tunneling from one vacuum $(\phi=0)$ to the neighboring one $(\phi=\pi)$ is dominated by instantons and evaluated to exponential accuracy by

$$
e^{-S_{s c}}=e^{-\int L_{s}^{e} d \tau}
$$

where

$$
L_{s}^{e}=\frac{m(\phi)}{2}\left(\frac{d \phi}{d \tau}\right)^{2}-i \alpha m(\phi) \frac{d \phi}{d \tau}+V(\phi)-\frac{m(\phi)}{2} \alpha^{2}
$$

is the Euclidean Lagrangian with the imaginary time $\tau=i t$. The imaginary part (the second term) in $L_{s}^{e}$ induced by the Zeeman term becomes a phase in eq.(6)

$$
e^{-S_{s c}}=e^{-\tilde{S}_{s c}} e^{i \theta_{s}}
$$

where $\tilde{S}_{s c}$ is the remaining action, and the phase derived with the semiclassical method is seen to be

$$
\theta_{s}=\int_{0}^{\pi} \alpha m(\phi) d \phi=\frac{\alpha \pi}{2 K_{1} \sqrt{1-\lambda}}
$$

which leads to the quantum phase interference between clockwise and anticlockwise tunnelings. Since we here emphasize the phase induced by the Zeeman energy term, the known phase term $s \frac{d \phi}{d \tau}$ (responsible for the spin parity effect) which we omitted in the Euclidean action should be understood. We now turn to the quantum theory of spin.

Following ref.[12] we start from the Schrödinger equation 


$$
\hat{H} \Phi(\phi)=E \Phi(\phi)
$$

The explicit form of the action of the spin operator on the function $\Phi(\phi)$ is seen to be

$$
\hat{S}_{x}=s \cos \phi-\sin \phi \frac{d}{d \phi}, \quad \hat{S}_{y}=s \sin \phi+\cos \phi \frac{d}{d \phi}, \quad \hat{S}_{z}=-i \frac{d}{d \phi}
$$

where the generating function $\Phi(\phi)$ is constructed in terms of the conventional spin functions of the $\hat{S}_{z}$ representation such as

$$
\Phi(\phi)=\sum_{m=-s}^{s} \frac{c_{m}}{\sqrt{(s-m) !(s+m) !}} e^{i m \phi}
$$

which obviously obeys the following boundary condition

$$
\Phi(\phi+2 \pi)=e^{2 \pi i s} \Phi(\phi)
$$

Thus we have periodic wave functions for integer spin s and antiperiodic functions for halfinteger s. The antiperiodic wave functions naturally give rise to the spin parity effect as we shall see. Substitution of the differential spin operators eq.(11) into eq.(10) yields

$$
\left[-K_{1}\left(1-\lambda \sin ^{2} \phi\right) \frac{d^{2}}{d \phi^{2}}-K_{2}\left(s-\frac{1}{2}\right) \sin 2 \phi \frac{d}{d \phi}+i \alpha \frac{d}{d \phi}+V(\phi)\right] \Phi(\phi)=E \Phi(\phi)
$$

with

$$
V(\phi)=K_{2} s^{2} \cos ^{2} \phi+K_{2} s \sin ^{2} \phi
$$

In the new variable $\mathrm{x}$ defined by

$$
x=\int_{0}^{\phi} \frac{d \phi^{\prime}}{\sqrt{1-\lambda \sin ^{2} \phi}}=F(\phi, k)
$$

which is the incomplete elliptic integral of the first kind with modulus $k^{2}=\lambda$, the trigonometric functions $\sin \phi$ and $\cos \phi$ become the Jacobian elliptic functions $\operatorname{sn}(\mathrm{x}), \mathrm{cn}(\mathrm{x})$ with the same modulus respectively. We then make use of the following transformation,

$$
\Phi(\phi(x))=d n^{s}(x) e^{i f(x)} \psi(x)
$$


where $d n(x)=\sqrt{1-\lambda s n^{2}(x)}$ is also a Jacobian elliptic function. Substituting eq.(17) into eq.(14) we obtain, after some tedious but not too complicated algebra, an equivalent Schrödinger equation with the desired Hamiltonian, i.e.

$$
\left\{K_{1}\left[-i \frac{d}{d x}+A(x)\right]^{2}+U(x)\right\} \psi(x)=E \psi(x)
$$

The function $\mathrm{f}(\mathrm{x})$ in the unitary transformation is determined by the requirement of gauge covariance and the scalar potential is required to be real. $f(x)$ is therefore defined by

$$
\frac{d f(x)}{d x}=\frac{-\alpha s}{K_{1} d n(x)}
$$

A gauge potential induced by the Zeeman energy term is found to be

$$
A(x)=-\frac{\alpha(2 s+1)}{2 K_{1} d n(x)}
$$

The scalar potential

$$
U(x)=\xi c d^{2}(x), \quad c d(x)=\frac{c n(x)}{d n(x)}
$$

is periodic with period $2 \mathcal{K}(k)$ and symmetric with respect to the coordinate origin $x=$ $o(U(x)=U(-x))$. The quantity $\mathcal{K}(k)$ is the complete elliptic integral of the first kind. The minima of the potential, namely, the vacua which have been shifted to zero by adding a constant, are located at $\pm(2 n+1) \mathcal{K}(k)$ with $n$ being an integer. The positions of potential peaks are at $\pm 2 n \mathcal{K}(k)$, and barrier height is

$$
\xi=K_{2} s(s+1)+\frac{\lambda \alpha^{2}}{4(1-\lambda) K_{1}}
$$

When $\alpha=0$, i.e. the Zeeman term in eq.(1) vanishes, the potential becomes exactly the same as that in ref.[15]. The shape of the scalar potential is not changed by the external magnetic field along the hard axis. In the new variable $\mathrm{x}$ the wave function $\Phi(\phi(x))$ is also periodic for integer s and antiperiodic for half-integer s with a period $4 \mathcal{K}(k)$ and the boundary condition of the wave function $\psi(x)$ is, however, determined by eqs.(17) and (19), i.e. 


$$
\psi(x+4 \mathcal{K}(k))=(-1)^{2 s} e^{i \frac{2 \alpha s \pi}{K_{1} \sqrt{1-\lambda}}} \psi(x)
$$

One should bear in mind from eq.(16) that $x=\mathcal{K}(k)$ corresponds to the original angle variable $\phi=\frac{\pi}{2}$. The boundary condition eq.(23) plays an important role in the following calculation of the tunneling splitting.

\section{TUNNELING SPLITTING AT THE GROUND STATE}

The tunneling between degenerate vacua (the case we consider here) results in the level splitting and is dominated by (vacuum) instantons which are nontrivial solutions of the Euclidean equation of motion with finite action. In the context of quantum mechanics the instanton may be visualized as a pseudoparticle moving between degenerate vacua under the barrier and has nonzero topological charge but zero energy. The tunneling splitting can be obtained from the transition amplitude between degenerate vacua which has a Euclidean path-integral representation. The first explicit calculation of the tunneling splitting in terms of the instanton method was carried out long ago for the double-well potential [16].

The Hamilton function corresponding to eq.(18) is

$$
H=\frac{1}{2 m}[P+A(x)]^{2}+U(x)
$$

where $m=\frac{1}{2 K_{1}}$ is the mass of the point-like particle. The Lagrangian is

$$
L=\frac{m}{2} \dot{x}^{2}-A(x) \dot{x}-U(x)
$$

With the Wick rotation $\tau=i t$ the Euclidean Lagrangian is seen to be

$$
L_{e}=\frac{m}{2} \dot{x}^{2}+i A(x) \dot{x}+U(x)
$$

In the above Euclidean Lagrangian and from now on $\dot{x}=\frac{d x}{d \tau}$ denotes the imaginary time derivative. The gauge potential $A(x)$ indeed does not affect the equation of motion which is

$$
m \ddot{x}=\frac{d U(x)}{d x}
$$


However, it leads to the phase interference which can be observed as the oscillation of tunneling splitting. This is exactly an $\mathrm{AB}$ effect in a generalized meaning. The instanton solution of eq.(27) can be found by direct integration and the result is

$$
x_{c}(\tau)=s n^{-1}(\tanh \omega \tau), \quad \omega^{2}=4 K_{1} \xi
$$

which is nothing but a kink configuration. The instanton starts from the vacuum $x_{i}=-\mathcal{K}(k)$ at $\tau=-\infty$ and reaches the centre of the potential barrier $(x=0)$ at $\tau=0$ and then arrives at the neighboring vacuum $x_{f}=\mathcal{K}(k)$ at $\tau=\infty$. The Euclidean action evaluated along the instanton trajectory is

$$
S_{c}=\int_{-\infty}^{\infty} L_{e}\left(x_{c}(\tau), \dot{x}_{c}(\tau)\right) d \tau=B-i(2 s+1) \theta_{s}
$$

where the first term

$$
B=\sqrt{\frac{\xi}{K_{2}}} \ln \frac{1+\sqrt{\lambda}}{1-\sqrt{\lambda}}
$$

reduces to the well known action [8,11, 14, 17 [19] when $\alpha=0$. The imaginary part leads to a phase in the Euclidean Feynman propagator which is $2 s+1$ times the semiclassical phase $\theta_{s}$.

To calculate the tunneling splitting, we start from the instanton induced transition amplitude,

$$
\left\langle x_{f}(\beta) \mid x_{i}(-\beta)\right\rangle=\sum_{m_{f}, n_{i}}\left\langle x_{f} \mid m_{f}\right\rangle\left\langle m_{f}\left|\hat{P}_{E} e^{-\beta \hat{H}}\right| n_{i}\right\rangle\left\langle n_{i} \mid x_{i}\right\rangle
$$

$\hat{P}_{E}$ is the projector onto the subspace of fixed energy [20] and $\left|n_{i}\right\rangle,\left|m_{f}\right\rangle$ are the excitations above two vacua lying on different sides of the barrier. The left hand side of eq.(31) has the path integral representation and is evaluated in the following. We consider the tunneling from initial vacuum $x_{i}=-\mathcal{K}(k)$ (corresponing to the original angle variable $\phi_{i}=-\frac{\pi}{2}$ ) to the neighboring one $x_{f}=\mathcal{K}(k)\left(\phi_{f}=\frac{\pi}{2}\right)$ for the fixed energy $E_{0}$ which is the degenerate ground state energy. The small tunneling splitting of the ground state is obtained from eq.(31) such that 


$$
\Delta E_{0} \sim\left|\frac{e^{2 \beta E_{0}}}{\beta} F \int_{-\mathcal{K}(k)}^{\mathcal{K}(k)} \mathcal{D} x e^{-\int_{-\beta}^{\beta} L_{e} d \tau}\right|
$$

where

$$
F=\frac{1}{\psi_{0}(\mathcal{K}(k)) \psi_{0}^{*}(-\mathcal{K}(k))}=\frac{e^{-i\left(\pi s+2 s \theta_{s}\right)}}{N}, \quad N=\psi_{0}\left(0_{f}\right) \psi_{0}\left(0_{i}\right)
$$

The second equality in F comes from the boundary condition of our wave function eq.(23) and $0_{i}, 0_{f}$ denote the coordinate origins of the local frames associated with each potential well. $\mathrm{N}$ is then a normalization constant calculated with the harmonic oscillator approximated wave function of the ground state. Substitution of the Lagrangian eq.(26) into the Feynman kernel in eq.(32) yields our interesting phase,

$$
\int \mathcal{D} x e^{-\int_{-\beta}^{\beta} L_{e} d \tau}=e^{i(2 s+1) \theta_{s}} G\left(x_{f}, \beta ; x_{i},-\beta\right)
$$

It is somewhat surprising that the quantum theory gives rise to $2 s+1$ times the semiclassical phase angle $\theta_{s}$ instead of just one. In Appendix 2 we explain the reason why the significant phase angle $2 s \theta_{s}$ can be missed in the semiclassical treatment of spin based on the large spin s limit. However, the additional phase, i. e. $2 s \theta_{s}$, in the Euclidean Feynman kernel is cancelled by the the phase of $\mathrm{F}$ in eq.(33) and does not affect the tunneling splitting of the ground state. We will see in the following section that the cancellation would not be exact for excited states and the additional phase has effect on the tunneling splitting

We should bear in mind that the above phase is obtained by an anticlockwise tunneling. The remaining Feynman kernel

$$
G=\int \mathcal{D} x e^{-\int_{-\beta}^{\beta} L_{e}^{\prime} d \tau}, \quad L_{e}^{\prime}=\frac{m}{2} \dot{x}^{2}+U(x)
$$

is independent of the direction of tunneling. For the clockwise tunneling from the same initial position $-\mathcal{K}(k)$ to the final position $-3 \mathcal{K}(k)$ the result is the same except with an opposite sign of the phase. Adding the contributions of clockwise and anticlockwise tunnelings we finally have the tunneling splitting expressed as

$$
\Delta E_{0} \sim \frac{e^{2 \beta E_{0}}}{\beta N}\left|\cos \left[\pi s+\theta_{s}\right]\right| G\left(x_{f}, \beta ; x_{i},-\beta\right)
$$


The tunneling kernel $\mathrm{G}$ in the one loop approximation, namely including the preexponential factor, can be calculated with the standard procedure. Before we give the final result, it is worthwhile to point out that in the evaluation of $\mathrm{G}$ the contributions from one instanton and one instanton plus the infinite number of instanton-anti-instanton pairs will be taken into account. However, the phase induced by the gauge potential for an instanton-anti-instanton pair vanishes. Thus the single instanton phase is factored out off the tunneling kernel. We have

$$
G \sim 2 N \beta e^{-2 \beta E_{0}} Q e^{-B}, \quad Q=2^{\frac{5}{2}}\left[\frac{\xi^{\frac{3}{2}} K_{1}^{\frac{1}{2}}}{(1-\lambda) \pi}\right]^{\frac{1}{2}}
$$

where $N=\frac{1}{\sqrt{2 \pi}}\left(\frac{\xi}{K_{1}}\right)^{\frac{1}{4}}$, and $E_{0}=\frac{\omega}{2}$ is the usual ground state energy of the harmonic oscillator. The tunneling splitting is thus [14]

$$
\Delta E_{0}=\left|\cos \left[\pi s+\theta_{s}\right]\right| 4 \Delta \varepsilon_{0}, \quad \Delta \varepsilon_{0}=Q e^{-B}
$$

When the external magnetic field vanishes $(\alpha=0)$ the tunneling splitting reduces exactly to the previous result [8,14] except that $s^{2}$ in the splitting amplitude of the semiclassical treatment is corrected as $s(s+1)$ by the quantum theory of spin. The well known spin parity effect (namely, the tuuneling splitting would be quenched for half-integer spin s) is recovered by the factor $|\cos \pi s|$ and is surely due to the antiperiodicity of the wave function in the quantum theory of spin. The tunneling splitting oscillates with the external field $\mathrm{h}$ and vanishes when

$$
s \pi+\theta_{s}=\pi\left[s+\frac{\mu_{B} g h}{2 K_{1} \sqrt{1-\lambda}}\right]=\left(n+\frac{1}{2}\right) \pi
$$

where $n$ is an integer. The oscillation period of the tunneling splitting with respect to the external field $\mathrm{h}$ is given by

$$
\Delta h=\frac{2 K_{1} \sqrt{1-\lambda}}{g \mu_{B}}
$$

To verify the validity of the tunneling splitting eq.(38) we compare the splitting value of eq.(38) as a function of the external magnetic field $\mathrm{h}$ with the numerical result obtained 
by performing a diagonalization of the Hamilton operator eq.(1). Adopting the data of the anisotropy contants given in ref.[4] such that $D=0.292 K, E=0.046 K$ and taking into account the relations between anisotropy constants $K_{1}, K_{2}$ and D, E, i.e. $K_{1}=D+E$, $K_{2}=D-E$, the oscillating amplitude of the tunneling splitting calculated from eq.(38) which begins from $6.286 \times 10^{-10} \mathrm{~K}$ for $s=10$ and increases with the magnetic field agrees with the numerical value of diagonalization perfectly. The period is $\Delta h=0.26 T$ which is substantially smaller than the experimental value $0.4 \mathrm{~T}$ [4. It has been pointed out that the discrepancies between experimental and theoretical results can be resolved by including

higher order terms of $\hat{S}_{z}$ and $\hat{S}_{x}$ in the Hamilton operator eq.(1) besides the quadratic terms [4].

\section{TUNNELING SPLITTING AND QUANTUM PHASE INTERFERENCE AT EXCITED STATES}

The quantum phase induced by the Zeeman term is manifestly computed from the Euclidean Feynman paths between two turning points which depend on energy. We now investigate the tunneling and related quantum phase interference at excited states. The starting point is again the transition amplitute of the barrier penetration projected onto the subspace of fixed energy, i.e.

$$
\sum_{m, n}<E_{n}^{f}\left|\hat{P}_{E} e^{-2 \beta \hat{H}}\right| E_{m}^{i}>=\int d x_{f} d x_{i} \psi_{E}^{*}\left(x_{f}\right) \psi_{E}\left(x_{i}\right) G\left(x_{f}, \beta ; x_{i},-\beta\right)
$$

from which the tunneling splitting is written as,

$$
\Delta E \sim \frac{e^{2 E \beta}}{\beta}\left|e^{-i s\left(\pi-2 \theta_{s}\right)} \int d \tilde{x}_{f} d \tilde{x}_{i} \psi_{E}\left(\tilde{x}_{f}\right) \psi_{E}\left(\tilde{x}_{i}\right) G\right|
$$

where $\tilde{x}_{i}=\mathcal{K}(k)+x_{i}, \tilde{x}_{f}=-\mathcal{K}(k)+x_{f}$ denote the coordinates in the local frames with origins at $-\mathcal{K}(k)$ and $\mathcal{K}(k)$ respectively. Thus the phase factor of our wave function $\psi_{E}$ can be factorized out. The tunneling at finite energy $\mathrm{E}$ is dominated by the periodic instanton 21,22 which satisfies the following integrated equation of motion, 


$$
\frac{m}{2} \dot{x}^{2}-U(x)=-E
$$

with periodic boundary condition. The periodic instanton is found to be

$$
x_{c}(\tau)=c d^{-1}\left(\sqrt{\frac{\left[\sin ^{-1} \operatorname{sn}(\tilde{\omega} \tau, \tilde{k})\right]^{2}\left(1-\eta^{2}\right)-\left(1-\lambda \eta^{2}\right)}{\lambda\left[\sin ^{-1} \operatorname{sn}(\tilde{\omega} \tau, \tilde{k})\right]^{2}\left(1-\eta^{2}\right)-\left(1-\lambda \eta^{2}\right)}}, \tilde{k}\right)
$$

where

$$
\tilde{\omega}=2 \sqrt{K_{1} \xi\left(1-\lambda \eta^{2}\right)}, \quad \eta=\sqrt{\frac{E}{\xi}}, \quad \tilde{k}^{2}=\frac{1-\eta^{2}}{1-\lambda \eta^{2}}
$$

The periodic instanton moves between two turning points $x_{ \pm}$depending on energy

$$
x_{ \pm}= \pm c d^{-1}(\eta, \tilde{k})
$$

When the energy tends to zero the periodic instanton reduces to the vacuum instanton of eq.(28). The Euclidean action evaluated along the periodic instanton is

$$
S_{c}=W+2 E \beta-i \theta_{E}
$$

where

$$
W=\int_{-\beta}^{\beta} m \dot{x}_{c}^{2} d \tau=2 \eta^{2} \sqrt{\frac{\xi}{K_{1}\left(1-\lambda \eta^{2}\right)}}\left[\Pi\left(\eta^{\prime 2}, \tilde{k}\right)-\mathcal{K}(\tilde{k})\right]
$$

with $\eta^{\prime 2}=1-\eta^{2}$, where $\Pi$ denotes the complete elliptic integral of the third kind. The tunneling phase for the anticlockwise tunneling (from $x_{-}$to $x_{+}$) is seen to be

$$
\theta_{E}=\int_{x_{-}}^{x_{+}} A\left(x_{c}\right) d x_{c}=\frac{(2 s+1) \alpha}{K_{1} \sqrt{1-\lambda}}\left[\tan ^{-1} \frac{\eta^{\prime}-\eta}{\eta^{\prime}+\eta}+\frac{\pi}{4}\right]
$$

which tends to the vacuum instanton phase when $E=0\left(\eta=0, \eta^{\prime}=1\right)$. The clockwise tunneling gives rise to the same result except for the phase with an opposite sign. Adding the two classes of the tunneling kernels the level splitting is seen to be

$$
\Delta E \sim \frac{e^{2 E \beta}}{\beta}\left|\cos \left(s \pi+\theta_{E}-2 s \theta_{s}\right)\right| I
$$

where 


$$
I=\int d \tilde{x}_{f} d \tilde{x}_{i} \psi_{E}\left(\tilde{x}_{f}\right) \psi_{E}\left(\tilde{x}_{i}\right) \tilde{G}
$$

The term $2 s \theta_{s}$ in eq.(50) comes from the boundary condition of $\psi$ eq.(23). The difference, i.e. $\theta_{E}-2 s \theta_{s}$, is not just a simple semiclassical phase $\theta_{s}$ in this case. The phase independent tunneling kernel $\tilde{G}$ is now evaluated with the help of the periodic instanton. Following the procedure in refs.[23] and [24] we take into account the contributions of the instanton and instanton plus the infinite number of pairs and compute the end point integrations with the help of WKB wave functions for $\psi_{E}$. A quite general formula for eq.(51) is

$$
I \sim 2 \beta e^{-2 E \beta}\left[\frac{1}{4 \mathcal{K}(\hat{k})}\right] e^{-W}, \quad \hat{k}^{2}=\frac{(1-\lambda) \eta^{2}}{1-\lambda \eta^{2}}
$$

The level splitting is then given by

$$
\Delta E=\left|\cos \left(s \pi+\theta_{E}-2 s \theta_{s}\right)\right| \frac{1}{\mathcal{K}(\hat{k})} e^{-W}
$$

For low lying excited states $\left(\eta<<1, \hat{k}<<1, \tilde{k}^{\prime}=\sqrt{1-\tilde{k}^{2}}<<1\right)$ the energy E may be replaced by harmonic oscillator approximated eigenvalues $E \rightarrow E_{n}=\left(n+\frac{1}{2}\right) \omega$. Expanding the complete elliptic integrals $\Pi\left(\eta^{\prime 2}, \tilde{k}\right), \mathcal{K}(\tilde{k})$ in $\mathrm{W}$ (eq.(48)) as power series of $\tilde{k}^{\prime}$ and $\mathcal{K}(\hat{k})$ in eq.(52) as power series of $\hat{k}$ we obtain after some tedious algebra the tunneling splitting of the nth excited state, i.e.

$$
\Delta E_{n}=\left|\cos \left(s \pi+\theta_{E_{n}}-2 s \theta_{s}\right)\right| 4 \Delta \varepsilon_{n}
$$

where

$$
\Delta \varepsilon_{n}=\frac{2^{3 n}}{n !(1-\lambda)^{n}}\left(\frac{\xi}{K_{1}}\right)^{\frac{n}{2}} \Delta \varepsilon_{0}
$$

In eq.(54) $\theta_{E_{n}}$ denotes the phase angle at nth excited state which is obtained from eq.(49) with replacing the energy E by $\left(n+\frac{1}{2}\right) \omega$. When $\alpha=0$ the tunelling splittings at excited states again coincide with the previous results [8,15] in terms of the semiclassical treatment of spin in large spin limit which means that the difference between $s^{2}$ and $s(s+1)$ can be neglected. 


\section{CONCLUSION}

On the basis of the UZ method for quantum spin systems we found that the Zeeman term of the external magnetic field along the hard axis for a biaxial spin particle indeed turns out to be a gauge potential in the point-particle-like Hamilton operator. The gauge potential does not affect the equation of motion but leads to quantum phase interference as an $\mathrm{AB}$ type effect in the spin tunneling. An additional phase angle $2 s \theta_{s}$ of the Euclidean action obtained by means of the quantum mechanical treatment of spin does not affect the tunneling splitting of the ground state but has effect on the tunneling splitting of excited states. In addition the splitting amplitude is modified by the quantum theory of spin. We present a formula of the tunneling splitting, eq.(54), as a function of the magnetic field, valid for low lying excited states, which for molecular clusters in which the total spin is only about ten is more accurate than the semiclassical treatment of spin for describing the quantum tunneling.

Acknowledgment: This work was supported by the National Natural Science Foundation of China under Grant Nos. 1967701 and 19775033. J.-Q. L. and D. K. P. also acknowledge support by the Deutsche Forschungsgemeinschaft.

\section{Appendix 1:Approximate spin-coordinate correspondance in the semiclassical treatment of spin}

In the conventional application of the spin coherent state technique, two canonical variables, $\phi$ and $p=s \cos \theta$ are adopted with the usual quantization

$$
[\phi, p]=i
$$

We show in the following that the spin-coordinate correspondence is only approximate up to order $0\left(s^{-3}\right)$. 
From the relation between the spin operators and the polar coordinate angles

$$
S_{x}=s \sin \theta \cos \phi, \quad S_{y}=s \sin \theta \sin \phi, \quad S_{z}=s \cos \theta
$$

the usual commutation relation of spin operators reads

$$
\left[S_{x}, S_{y}\right]=s^{2}[\sin \theta \cos \phi, \sin \theta \sin \phi]=s^{2} \sin \theta[\cos \phi, \sin \theta] \sin \phi+s^{2} \sin \theta[\sin \theta, \sin \phi] \cos \phi
$$

Using eq.(A1), one can prove the following relations

$$
[\sin \theta, \cos \phi]=A_{+} \cos \phi+i A_{-} \sin \phi, \quad[\sin \theta, \sin \phi]=A_{+} \sin \phi-i A_{-} \cos \phi
$$

with

$$
\begin{gathered}
A_{+}=\frac{1}{2}\left(\sqrt{1-(\cos \theta+\gamma)^{2}}+\sqrt{1-(\cos \theta-\gamma)^{2}}\right), \\
A_{-}=\frac{1}{2}\left(\sqrt{1-(\cos \theta+\gamma)^{2}}-\sqrt{1-(\cos \theta-\gamma)^{2}}\right)
\end{gathered}
$$

where $\gamma=\frac{1}{s}$. Substituting eq.(A4) into eq.(A3), one has

$$
\left[S_{x}, S_{y}\right]=-i s^{2} \sin \theta A_{-}=i \gamma s^{2} \cos \theta+0\left(\gamma^{3}\right)
$$

i.e.

$$
\left[S_{x}, S_{y}\right]=i S_{z}+0\left(s^{-3}\right)
$$

which implies that the usual commutation relation holds only in the large spin limit.

\section{Appendix 2: Recovering the semiclassical phase in the large s limit}

To understand the reason why the phase angle $2 s \theta_{s}$ is missed in the semiclassical treatment of spin we consider the following Schrödinger equation obtained without the unitary transformation $e^{i f(x)}$ in the transformation eq.(17) for our spin sytem: 


$$
\left[-K_{1} \frac{d^{2}}{d x^{2}}+i \frac{\alpha}{d n(x)} \frac{d}{d x}-i s \alpha \lambda \frac{\operatorname{sn}(x) c n(x)}{d n^{2}(x)}+U_{s}(x)\right] \psi(x)=E \psi(x), \quad U_{s}(x)=K_{2} s(s+1) c d^{2}(x)
$$

The Hamilton operator can be written as

$$
\hat{H}_{s}=K_{1}\left[-i \frac{d}{d x}-\tilde{A}(x)\right]^{2}-i\left(s+\frac{1}{2}\right) \lambda \alpha \frac{\operatorname{sn}(x) c n(x)}{d n^{2}(x)}+\tilde{U}_{s}(x)
$$

The gauge potential

$$
\tilde{A}(x)=\frac{\alpha}{2 K_{1} d n(x)}
$$

leads exactly to the semiclassical phase angle $\theta_{s}$, while the scalar potential which contains an imaginary part is ill-defined. In the large s limit one might neglect the imaginary part in comparison with the term $U_{s}(x)$ and then has the Hamilton operator given by

$$
\hat{H}_{s}=K_{1}\left[-i \frac{d}{d x}-\tilde{A}(x)\right]^{2}+\tilde{U}_{s}(x), \quad \tilde{U}_{s}(x)=K_{2} s(s+1) c d^{2}(x)-\frac{\alpha^{2}}{4 K_{1} d n^{2}(x)}
$$

The final Hamiltonian

$$
\tilde{H}_{s}=\frac{1}{2 m}[p-\tilde{A}(x)]^{2}+\tilde{U}_{s}(x), \quad m=\frac{1}{2 K_{1}}
$$

is the counterpart of the effective Hamiltonian $H_{s}$ of eq.(3). The corresponding Euclidean Lagrangian is

$$
\tilde{L}_{s}^{e}=\frac{m}{2} \dot{x}^{2}+i \tilde{A}(x) \dot{x}+\tilde{U}_{s}(x)
$$




\section{REFERENCES}

[1] A. J. Leggett, S. Chakravarty, A. T. Dorsey, M. P. A. Fisher, A. Garg, and W. Zwerger, Rev. Mod. Phys. 59, 1 (1987)

[2] Proceedings of the Meeting on Quantum Tunneling of Magnetization-QTM'94, edited by L. Gunther and B. Barbara, Vol. 301 of NATO ASI Series (Kluwer, Dordrecht, 1995).

[3] A. Garg, Phys. Rev. Lett. 71, 4249(1993).

[4] W. Wernsdorfer and R. Sessoli, Science, 284, 133 (1999)

[5] D. Loss, D. P. DiVincenzo, and G. Grinstein, Phys. Rev. Lett., 69, 3232 (1992).

[6] E. M. Chudnovsky, and D. P. DiVincenzo, Phys. Rev. B48, 10548 (1993).

[7] N. V. Prokof'ev, and P. C. E. Stamp, J. Phys. Condens. Matter 5, L663 (1993).

[8] J. -Q. Liang, H. J. W. Müller-Kirsten, and J. -G. Zhou, Z. Physik. B102, 525 (1997).

[9] J. -Q. Liang, L. Maharana, and H. J. W. Müller-Kirsten, to appear in Physica B.

[10] A. Garg, Europhys. Lett. 22, 209 (1993).

[11] S. P. Kou, J. Q. Liang, Y. B. Zhang, and F. C. Pu, Phys. Rev. B59, 11792 (1999).

[12] V. V. Ulyanov and O. B. Zaslavskii, Phys. Rep. 216, 179 (1992)

[13] A. Perelomov, Generalized Coherent States and Their Applications (Springer, Heidelberg 1986).

[14] J. -Q. Liang, Y. -B. Zhang, H. J. W. Müller-Kirsten, Jian-Ge Zhou, Z. Zimmerschied, and F. -C. Pu, Phys. Rev. B57, 529 (1998).

[15] Y. -B. Zhang, J. -Q. Liang, H. J. W. Müller-Kirsten, S. -P. Kou, X. -B. Wang and F. -C. Pu, cond-mat/9902066, to appear in Phys. Rev. B.

[16] E. Gildener and A. Patrascioiu, Phys. Rev. D16, 423 (1977). 
[17] M. Enz, and R. Schilling, J. Phys. C19, 1765 (1988).

[18] M. Enz, and R. Schilling, J. Phys. C19, L711 (1988).

[19] E. M. Chudnovsky, and L. Gunther, Phys. Rev. Lett., 60, 661 (1988).

[20] A. N. Kuznetsov, and P. G. Tinyakov, Phys. Lett., B406, 76 (1997).

[21] N. S. Manton and T. S. Samols, Phys. Lett. B207, 179 (1988).

[22] J. -Q. Liang, H. J. W. Müller-Kirsten, and D. -H. Tchrakian, Phys. Lett. B282, 105 (1992).

[23] J.-Q. Liang and H. J. W. Müller-Kirsten, Phys. Rev. D45, 4685 (1992).

[24] J.-Q. Liang and H. J. W. Müller-Kirsten, Phys. Rev. D51, 718 (1995). 